\title{
Dexamethasone combined with berberine is an effective therapy for bleomycin-induced pulmonary fibrosis in rats
}

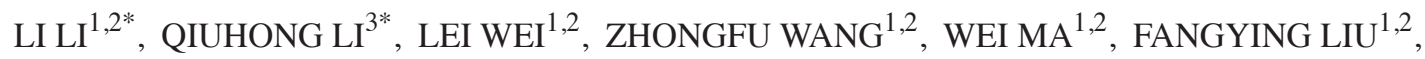 \\ YANHUA SHEN $^{1,2}$, SHANFANG ZHANG ${ }^{1,2}$, XIULIAN ZHANG ${ }^{1,2},{\text { HUIPING } \text { LI }^{3} \text { and YECHANG QIAN }}^{1,2}$ \\ ${ }^{1}$ Department of Respiratory Disease, Baoshan Branch, Shuguang Hospital, Shanghai University of Traditional \\ Chinese Medicine, Shanghai 200431; ${ }^{2}$ Department of Respiratory Disease, Baoshan District Hospital of Integrated Traditional \\ Chinese and Western Medicine, Shanghai 201900; ${ }^{3}$ Department of Respiratory Medicine, Shanghai Pulmonary Hospital, \\ School of Medicine, Tongji University, Shanghai 200433, P.R. China
}

Received December 13, 2017; Accepted September 13, 2018

DOI: $10.3892 /$ etm.2019.7861

\begin{abstract}
Pulmonary fibrosis (PF) is a heterogeneous pathological process in lung tissues with a considerable mortality rate. Currently, combination therapy represents an effective approach to treat PF. Dexamethasone (Dxs) and berberine (BBR) are widely applied to inhibit the progression of PF. Dxs plus penehyclidine hydrochloride or alfacalcidol have been reported more effective in therapy compared with any single drug treatment. However, whether Dxs plus BBR induces an increased antifibrotic effect remains unknown. The current study aimed to evaluate the therapeutic effect of BBR plus Dxs in bleomycin (BLM)-induced PF. A PF model in rats was established and rats were divided into control, BLM, BBR, Dxs and BBR plus Dxs groups ( $\mathrm{n}=9$ /group). On days 3, 7 and 14, blood samples were collected from the eyes of the rats ( $n=6 /$ group). CXC chemokine ligand 14 (CXCL14), collagen I, collagen III, matrix metalloproteinase (MMP)2 and MMP9 serum levels were measured by ELISA. On day 14, all rats were sacrificed. Hematoxylin and eosin analysis, Masson staining and hydroxyproline (Hyp) assessment were performed to observe histopathological changes and collagen deposition. mRNA and protein levels of CXCL14, CXC chemokine receptor 4
\end{abstract}

Correspondence to: Dr Yechang Qian, Department of Respiratory Disease, Baoshan District Hospital of Integrated Traditional Chinese and Western Medicine, 181 You Yi Road, Baoshan, Shanghai 201900, P.R. China

E-mail: qianyechang2@163.com

Dr Huiping Li, Department of Respiratory Medicine, Shanghai Pulmonary Hospital, School of Medicine, Tongji University, 507 Zheng Min Road, Yangpu, Shanghai 200433, P.R. China

E-mail: liw2013@126.com

${ }^{*}$ Contributed equally

Key words: pulmonary fibrosis, bleomycin, berberine plus dexamethasone, CXC chemokine ligand 14, matrix metalloproteinase 2 , matrix metalloproteinase $9, \operatorname{Smad} 2 / 3$
(CXCR4), collagen I/III, $\alpha$-smooth muscle actin ( $\alpha$-SMA), MMP2/9 and phosphorylated-Smad 2/3 in lung tissue were further evaluated. Similar effects in preventing lung damage were observed histopathologically for Dxs and BBR compared with the BLM group. These treatments further reduced levels of Hyp, CXCL14, CXCR4, collagen I/III, MMP2/9, $\alpha$-SMA and p-Smad 2/3. The combination of Dxs and BBR exhibited increased effectiveness compared with the single treatments. Results further suggested that antifibrotic mechanisms were involved in inhibiting CXCL14 and MMP2/MMP9 expression, and preventing the activation of Smad2/3 and hedgehog signaling pathways. The combined use of Dxs and BBR may represent a potential therapeutic approach for PF.

\section{Introduction}

Pulmonary fibrosis (PF) is a heterogeneous disorder of lung interstitial tissue (1). The process of PF is complex and following lung injury, inflammatory cells migrate to the injured site via chemokine gradients (2). Recruited leukocytes release cytokines and chemokines, which increase levels of local profibrotic interleukin (IL)-13, IL-4 and transforming growth factor (TGF)- $\beta$ (2). Subsequently, bone marrow-derived fibrocytes or resident fibroblasts proliferate and differentiate into myofibroblasts, leading to excessive extracellular matrix (ECM) synthesis, tissue reshaping and fibrotic lesions (3). Etiologies of PF vary and include chemicals (airborne contaminants and toxic components from the smoking), organic or inorganic dust, radiation and trauma (4). With persisting stimulation or failure to regulate wound repair, fibrosis can develop at any stage (5). Currently, idiopathic PF (IPF) is thought to be one of the most common form of PF, with median survival times of 3-5 years following diagnosis (6). Exploring novel and effective treatments for this condition is of great interest.

Molecular pathways engaged in PF pathogenesis have not been fully elucidated and are potentially multifactorial (7). Matrix metalloproteinase (MMP)2 and MMP9, also known as gelatinases, are secreted by various types of cells in the lungs (4). MMP2/9 are vital in digesting collagens IV/V and gelatin in the basement membrane, leading to ECM breakdown and migration of cells adherent to the interstitial ECM (4). In 
addition, imbalance of synthesis and degradation of ECM components contributes to airway remodeling in the context of PF (4). Furthermore, chemokine gradients are required for trafficking of circulating leukocytes and fibrocytes to the lung tissue. CXC chemokine receptor 4 (CXCR4) and its ligand 14 (CXCL14), serve a supporting role in PF development and are involved in therapeutic treatment regiments $(8,9)$. TGF- $\beta 1$ is essential in promoting fibroblast-myofibroblast transformation with enhancing ECM synthesis that contributes to PF (10). TGF- $\beta 1$ downstream signaling effects are executed by $\mathrm{Smad} 2 / 3$ (11). Therapeutic interventions based on these endogenous regulatory mechanisms may provide potential antifibrotic drugs or strategies to block PF.

Combination therapy is an attractive and promising method to prevent PF (8). Dexamethasone (Dxs) is widely used as an antifibrotic agent due to its protection of the lungs against fibrosis by inhibiting the production of inflammatory mediators (9). Berberine (BBR), a known non-toxic natural agent, inhibits nuclear factor- $\kappa \mathrm{B}$ proinflammatory and profibrotic mediators in bleomycin (BLM)-induced PF and is used as an alternative treatment counteracting PF (10). Reports demonstrated that Dxs plus penehyclidine hydrochloride (12) or alfacalcidol (13) attenuate PF more effectively compared with either single treatment. However, whether Dxs plus BBR has a better therapeutic effect in PF compared with the single treatments remains unknown. In the present study, a BLM-induced rat PF model was successfully constructed. The effect of Dxs plus BBR treatment on preventing lung damage and collagen deposition at histological levels was evaluated. In addition, the combination effects of Dxs plus BBR on hydroxyproline (Hyp), CXCL14, MMP2/9 and $\alpha$-smooth muscle actin ( $\alpha$-SMA) expression and on activation of the Smad2/3 signaling pathway were assessed. The present study suggested that the combination of Dxs with BBR presents an effective approach to prevent PF.

\section{Materials and methods}

Preparation of $P F$ rat model and treatments. A total of 30 male Wistar albino rats, aged 8-10 week old, weighing 180-220 g were purchased from Shanghai SIPPR-Bk Laboratory Animal Co. Ltd. The rats were acclimatized at $25^{\circ} \mathrm{C}$ with 12 -h light/dark cycles with relative humidity between $40-70 \%$ for a week, and had free access to food and water as previously reported (11). Following acclimation, rats were randomly divided into control, BLM, Dxs, BBR and Dxs plus BBR groups ( $=6 /$ group). PF was established through a single endotracheal injection of BLM (5 mg/kg; Zhejiang Hisun Pharmaceutical Co., Ltd.) in all groups except the control (11). Following 24 h, Dxs, BBR and Dxs plus BBR preventive groups were administered Dxs (3 mg/kg/day, Sigma Chemical Co.; Merck KGaA) (12), BBR (200 mg/kg/day, Aladdin Reagent Co., Ltd.) (11) or Dxs (3 mg/kg/day) plus BBR (200 mg/kg/day), respectively, by intraperitoneal injection for 14 successive days. The control and BLM groups were simultaneously treated with saline ( $2 \mathrm{ml} / \mathrm{kg} /$ day).

To study the involvement of the hedgehog $(\mathrm{Hh})$ signaling pathway in the effect of Dxs and BBR treatment, another three groups of rats were randomly divided into BLM, Dxs plus BBR and Dxs+BBR+purmorphamine groups (n=6/group) without a separate untreated control or a purmorphamine control. The BLM and Dxs plus BBR groups were established as mentioned above. Animals of the Dxs+BBR+purmorphamine group were BLM-induced and then intraperitoneally injected with Dxs (3 mg/kg/day) (12), BBR (200 mg/kg/day) (11) and purmorphamine $(0.69 \mathrm{mg} / \mathrm{kg} /$ day, Aladdin Reagent Co., Ltd.) (14) for 14 consecutive days. Rats were euthanized with an overdose of pentobarbital $(200 \mathrm{mg} / \mathrm{kg})$ and lung tissues were collected, prepared for histology evaluation and/or stored at $-80^{\circ} \mathrm{C}$ for western blot analysis.

Histology evaluation. The lung tissues from all rats were collected, routinely fixed in $10 \%$ buffered formalin at $4^{\circ} \mathrm{C}$ for $48 \mathrm{~h}$, dehydrated in a graded ethanol series $(50,70,85$, 95 and $100 \%$ ), cleared in xylene and embedded in paraffin. Sections of $4-\mu \mathrm{m}$ thickness were cut then deparaffinized. Hematoxylin and eosin (H\&E) staining was performed to observe histological changes. Briefly, the lung sections were stained with eosin (cat. no. 714094; BASO Diagnostic, Inc.) for $1 \mathrm{~min}$ at room temperature, rinsed with running water for $15 \mathrm{~min}$ and then dyed with hematoxylin (cat. no. 714094; BASO Diagnostic, Inc.) for $5 \mathrm{~min}$ at room temperature eosin another. Masson's trichrome staining was performed using Masson's trichrome kits (Beijing Leagene Biotechnology Co., Ltd.) to measure the density of collagen fibers according to the manufacturer' instruction. The degree of lung fibrosis was assessed by infiltration of inflammatory cells, thickness of the alveolar walls and severity of the collagen deposition. Histology evaluation and Masson's trichrome staining were performed with the same tissue positioning. Images of $\mathrm{H} \& \mathrm{E}$ and Masson's trichrome staining were obtained in the same field of vision and obtained using a light microscope (Eclipse Ni-E; Nikon Corporation) under x 200 magnification. For each lung tissue section, three fields were randomly selected for imaging.

Hydroxyproline (Hyp) assessment. Lung tissue samples (30-100 mg wet weight) were lysed in radioimmunoprecipitation assay lysis buffer (JRDUN Bio. Co., Ltd.) at $4^{\circ} \mathrm{C}$ for $30 \mathrm{~min}$. The lysates were centrifuged at $3,500 \mathrm{x}$ g at $4^{\circ} \mathrm{C}$ for $10 \mathrm{~min}$, and total protein levels in the supernatants were quantified using the BCA Protein assay kit (cat. no. PICPI23223; Thermo Fisher Scientific, Inc.). Hyp content in lung tissue extract was evaluated using a hydroxyproline assay kit (cat. no. A030-2; Nanjing Jiancheng Bioengineering Institute) according to the manufacturer's instructions and calculated using a microtiter plate reader (BioTek Instruments, Inc.) at a wavelength of $550 \mathrm{~nm}$.

Enzyme-linked immunosorbent (ELISA) analysis. On days 3, 7 and 14 , rats were anesthetized with $10 \%$ of chloral hydrate $(400 \mathrm{mg} / \mathrm{kg}$ body weight; intraperitoneal injection; Sinopharm Chemical Reagent Co., Ltd.). No signs of peritonitis were observed. Blood samples were extracted from the eyes then centrifuged at $3,000 \mathrm{x}$ g at $4^{\circ} \mathrm{C}$ for $5 \mathrm{~min}$ to separate the serum. ELISA was conducted to assess serum CXCL14, collagen I, collagen III, MMP2 and MMP9 content according to the manufacturer's protocols (Xin-Yu Biotechnology Pharmaceutical Co., Ltd.). The absorbance value (at $450 \mathrm{~nm}$ ) was recorded using a microplate reader (Bio-Rad Laboratories, 
Inc.). The catalogue numbers of ELISA kits used to quantify CXCL14, collagen I, collagen III, MMP2 and MMP9 were EK-3394, хуH142, хуH144, xy-1706E and bsk00125, respectively.

Reverse transcription-quantitative polymerase chain reaction $(R T-q P C R)$ analysis. Total RNA from lung tissues was obtained using TRIzol reagent (cat. no. 1596-026; Invitrogen; Thermo Fisher Scientific, Inc.). cDNA was obtained using the RevertAid First Stand cDNA Synthesis kit (cat. no. \#K1622; Fermentas; Thermo Fisher Scientific, Inc.) with following conditions for $60 \mathrm{~min}, 85$ for $5 \mathrm{~min}$ then $4^{\circ} \mathrm{C}$ for $5 \mathrm{~min}$. The prepared cDNA was stored at $-20^{\circ} \mathrm{C}$ and used for the next step. qPCR was conducted using the SYBR Green mix (cat. no. \#K0223; Thermo Fisher Scientific, Inc.). GAPDH was used as endogenous control. Primer sequences were as follows: CXCL14 (83 bp), forward, 5'-AGTGTAAGT GTTCCCGGAAGG-3' and reverse, 5'-GCAGTGTGGGTA CTTTGGCTT-3'; CXCR4 (125 bp), forward, 5'-GAAGTG GGGTCTGGAGACTAT-3' and reverse, 5'-TTGCCGACT ATGCCAGTCAAG-3'; collagen I (178 bp), forward, 5'-GCT GACCTTCCTGCGCCTAATG-3' and reverse, 5'-GGTGCT GTAGGTGAAGCGACTG-3'; collagen III (234 bp), forward, 5'-ATGCCCACAGCCTTCTAC-3' and reverse, 5'-CCCACT CCAGACTTGACATC-3'; $\alpha$-SMA (104 bp), forward, 5'-CCC AGACATCAGGGAGTAATGG-3' and reverse, 5'-TCTATC GGATACTTCAGCGTCA-3'; MMP2 (117 bp), forward, 5'-ATGCCATCCCTGATAACC-3' and reverse, 5'-ACTTCA CGCTCTTGAGAC-3'; MMP9 (203 bp), forward, 5'-AGG GAGATGCCCATTTCG-3' and reverse, 5'-GCCGTCCTT ATCGTAGTCAG-3'; and GAPDH (197 bp), forward, 5'-CTG CCCAGAACATCATCC-3' and reverse, 5'-CTCAGATGC CTGCTTCAC-3'. The amplification condition were as follows: $95^{\circ} \mathrm{C}$ for $10 \mathrm{~min}$; 40 cycles of $95{ }^{\circ} \mathrm{C}$ for $15 \mathrm{sec}, 60^{\circ} \mathrm{C}$ for $45 \mathrm{sec}$ and then $95^{\circ} \mathrm{C}$ for $15 \mathrm{sec}$. The $2^{-\Delta \Delta \mathrm{Cq}}$ method was used for relative quantification (15).

Western blot analysis. Lung tissue samples were homogenized using radioimmunoprecipitation assay buffer (cat. no. R0010; Beijing Solarbio Science \& Technology Co., Ltd.) and centrifuged $\left(12,000 \times \mathrm{g} ; 10 \mathrm{~min} ; 4^{\circ} \mathrm{C}\right)$. Total protein contents were quantified using a bicinchoninic acid protein assay kit (cat. no. PICPI23223; Thermo Fisher Scientific, Inc.). Western blotting was performed as previously described (11). Briefly, $25 \mu \mathrm{g}$ of total protein were loaded per lane and isolated on $15 \%$ SDS-PAGE, then transferred onto nitrocellulose (NC) membranes (cat. no. HATF00010; EMD Millipore). Following blocking with $5 \%$ nonfat milk in TBST buffer $(50 \mathrm{mM}$ Tris [pH 7.4], $100 \mathrm{mM} \mathrm{NaCl}, 0.1 \%$ Tween-20) for $1 \mathrm{~h}$ at $25^{\circ} \mathrm{C}$, $\mathrm{NC}$ membranes were incubated with the primary antibodies. Antibodies were diluted in blocking solution (5\% nonfat milk) prior to use. The following antibodies were used: Anti-CXCR4 (1:2,000; cat. no. Ab181020), anti-CXCL14 (1:1,000; cat. no. Ab137541), anti-MMP2 (1:2,000; cat. no. Ab37150), anti-MMP9 (1:2,000; cat. no. Ab38898), anti-collagen I (1:1,000; cat. no. Ab6308), anti-collagen III (1:1,000; cat. no. Ab7778), anti- $\alpha$-SMA (1:300; cat. no. Ab5694) and anti-phosphorylated (p)-Smad2/3 (1:500; cat. no. Ab63399; all Abcam); and anti-Smad2/3 (1:1,000; cat. no. \#8685) and anti-GAPDH (1:2,000; cat. no. \#5174; all Cell Signaling
Technology, Inc.) at $4^{\circ} \mathrm{C}$ overnight. Next membranes were incubated with horseradish peroxidase-conjugated secondary antibodies (1:1,000; cat. nos. A0208, A0181 and A0216; Beyotime Institute for Biotechnology) for $1 \mathrm{~h}$ at $25^{\circ} \mathrm{C}$. Bands were visualized and quantified using an enhanced chemiluminescence system (Amersham; GE Healthcare). GAPDH served as internal control and $\mathrm{Smad} 2 / 3$ was used in the evaluation of the phosphorylation of Smad2/3. Predicted band sizes for collagen I, collagen III, CXCR4, CXCL14, MMP2, MMP9, $\alpha$-SMA, p-Smad2/3, Smad2/3 and GAPDH were 130, $138,39,13,72,89,42,48,53$ and $37 \mathrm{kDa}$, respectively and the molecular weights were 133, 140, 44, 14, 72, 92, 41, 47, 54 and $36 \mathrm{kDa}$, respectively. Band density was quantified with ImageJ software version 1.7 (National Institutes of Health).

Statistical analysis. Each experiment was independently performed $\geq 3$ times. Data are presented as the mean \pm standard error of the mean. Student's t-test was used to compare two groups and one-way analysis of variance with post-hoc Tukey's test was used to compare multiple groups. $\mathrm{P}<0.05$ was considered to indicate a statistically significant difference.

\section{Results}

Dxs plus BBR attenuates BLM-induced histological changes. Histological changes in lung tissues were analyzed by $\mathrm{H} \& \mathrm{E}$ staining. As presented in Fig. 1, the BLM-treated group exhibited marked morphologic changes compared with the control, including: i) Serious inflammatory infiltration, with fibroblasts in the lung interstitium; ii) extensive collapsed alveoli, a disappearing alveolar space and abundant cord-type fibrous tissues; and iii) a thickened alveolar interval and increased pulmonary interstitial substances. Dxs or BBR treatment decreased BLM-induced lung damage as follows: i) Decreased phagocytic infiltration and inflammatory cells, and proliferated fibroblasts observed in the lung interstitium; ii) fewer damaged alveoli; and iii) slightly thickened alveolar spacing and less pulmonary interstitial accumulation. Effects of Dxs plus BBR were better compared with Dxs or BBR treatment alone. Pulmonary damage was markedly reduced to normal levels, demonstrating that the combination of Dxs and BBR presented more effective in inhibiting PF at a histological level.

Dxs plus BBR reduces collagen deposition and Hyp content. Using Masson's staining (Fig. 2), it was evident that BLM (highlighted by square area) induced severe collagen deposition and alveolar thickening in lung tissues compared with the control. Dxs or BBR treatment markedly inhibited collagen deposition when compared with the BLM group. No differences in collagen deposition were observed between the control and the Dxs plus BBR groups.

Hyp content in the lung tissues, a representative marker for collagen deposition, was further measured. As summarized in Fig. 3, BLM significantly increased Hyp levels compared with the control $(\mathrm{P}<0.01)$. Dxs or BBR treatment had a beneficial outcome, significantly reducing the Hyp levels compared with the BLM group $(\mathrm{P}<0.01)$. These observations are in line with the Masson's analysis. Combination of Dxs and BBR induced a significantly greater inhibitory effect on collagen accumulation compared with Dxs or BBR single treatment $(\mathrm{P}<0.01)$. 


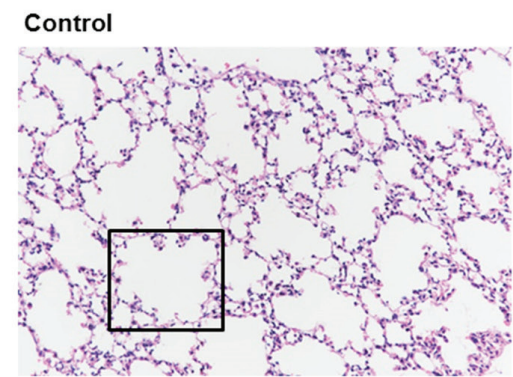

BLM

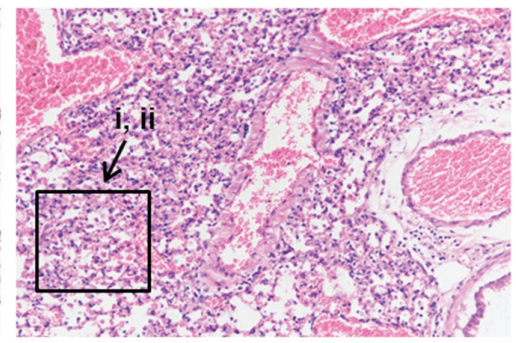

Dxs

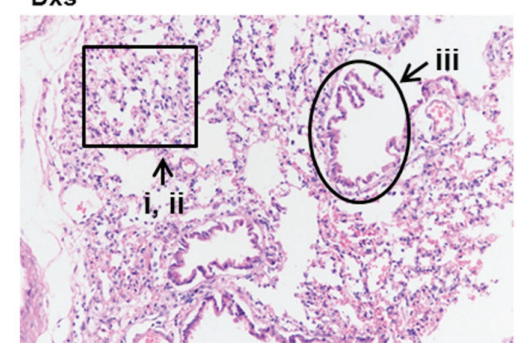

BBR

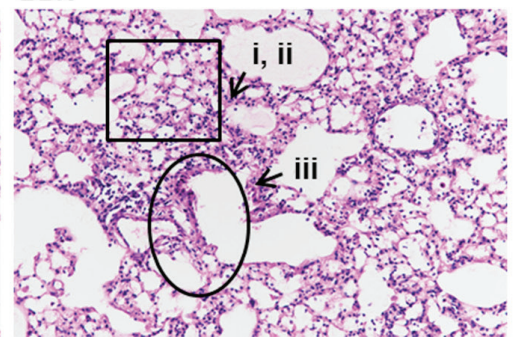

BLM

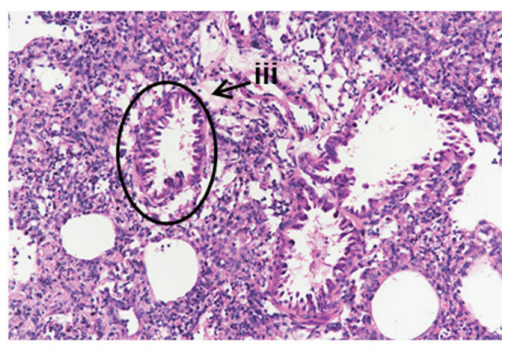

Dxs + BBR

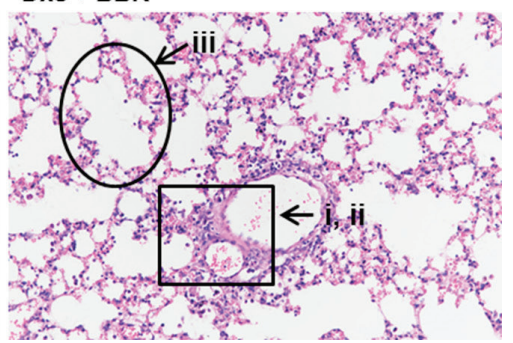

Figure 1. Histological evaluation of lung tissues from a rat model with pulmonary fibrosis. Hematoxylin and eosin staining was performed on tissue sections of control animals, BLM-induced rats with PF and BLM-induced animals treated with Dxs, BBR or Dxs plus BBR and sections were viewed using a light microscope (magnification, x200). Control group represents normal lung tissue with thin lined alveolar septa and well-architected alveolar space (the square area). BLM treatment induced distorted lung morphologies, including collapsed alveolar spaces with inflammatory exudates (i and ii) and wider and thicker alveolar intervals (iii). Dxs and BBR treatment improved lung damage, exhibiting fewer inflammatory infiltrations (i and ii) and less alveolar thickening (iii). Dxs plus BBR treatment left lung tissues comparable to the control. BLM, bleomycin; Dxs, dexamethasone; BBR, berberine.

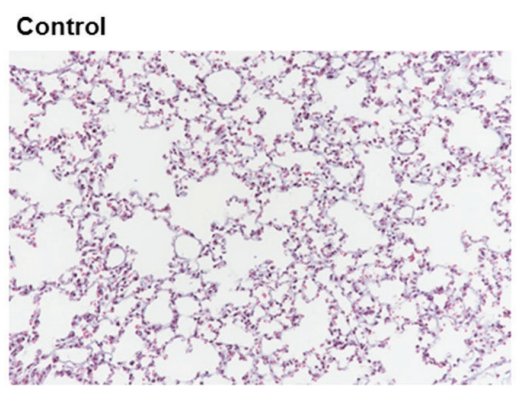

BLM
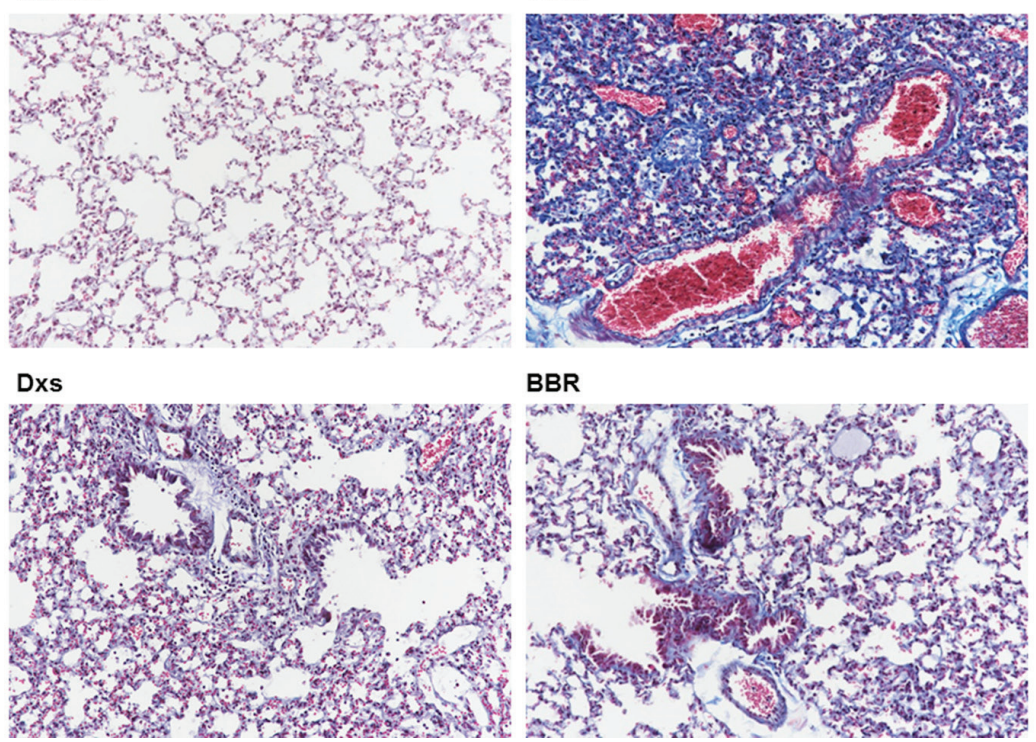

BBR

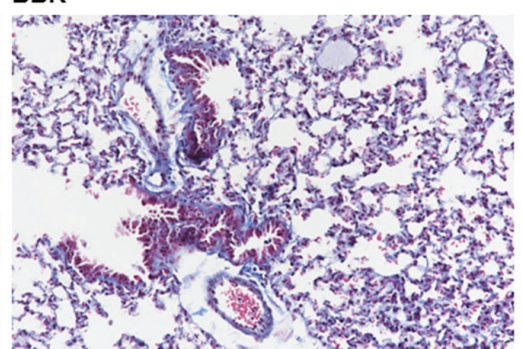

BLM

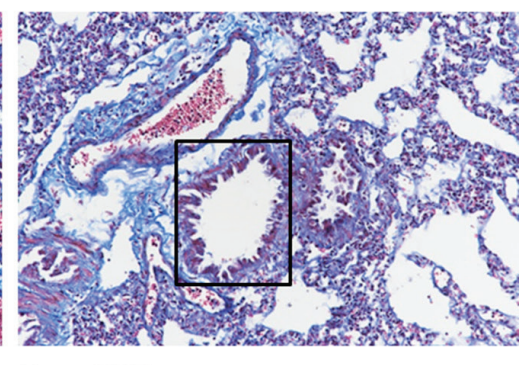

Dxs + BBR

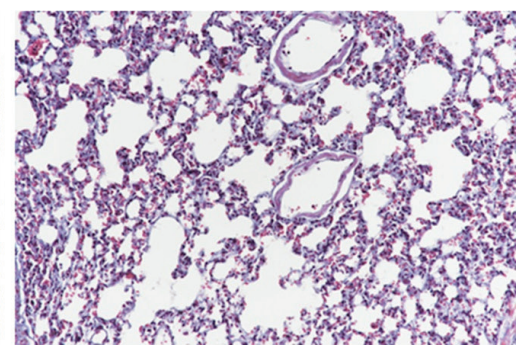

Figure 2. Collagen assessment via Masson's trichrome staining in lung tissues from a rat model with pulmonary fibrosis. Staining was performed on tissue sections of control animals, BLM-induced rats with PF and BLM-induced animals treated with Dxs, BBR or Dxs plus BBR and sections were viewed using a light microscope (magnification, x200). Collagen was dyed blue while muscle actin was red. Control and Dxs plus BBR exhibited minimal collagen accumulation in lung tissues. The BLM group demonstrated dense depositions of collagen and few muscle actin with the square area highlighting enhanced alveolar thickening with considerable collagen. The effects were alleviated with Dxs or BBR treatment. BLM, bleomycin; Dxs, dexamethasone; BBR, berberine.

Dxs plus BBR reduces levels of fibrogenesis-associated makers. To assess the antifibrotic effect of Dxs plus BBR treatment at molecular level, collagen I/III and differentiation-dependent $\alpha$-SMA levels in serum and lung tissues were measured. p-Smad2/3 was further evaluated via measuring the ratio of $\mathrm{p}-\mathrm{Smad}$ 2/3: Total Smad2/3 levels. As presented in Fig. 4, BLM significantly increased collagen I/III, $\alpha$-SMA and $\mathrm{p}-\mathrm{Smad} 2 / 3$ levels compared with the control $(\mathrm{P}<0.01)$.
This effect was significantly alleviated by Dxs or BBR single treatment $(\mathrm{P}<0.01)$. No significant differences in decreasing fibrogenesis-associated markers were observed between Dxs and BBR single treatment groups. Dxs plus BBR treatment significantly decreased collagen I/III (all $\mathrm{P}<0.05$, except for serum collagen I at day 3$), \alpha-\operatorname{SMA}(\mathrm{P}<0.05)$ and $\mathrm{p}-\mathrm{Smad} 2 / 3$ $(\mathrm{P}<0.01)$ levels compared with the Dxs or BBR groups. The data suggest that Dxs plus BBR was effective in preventing 


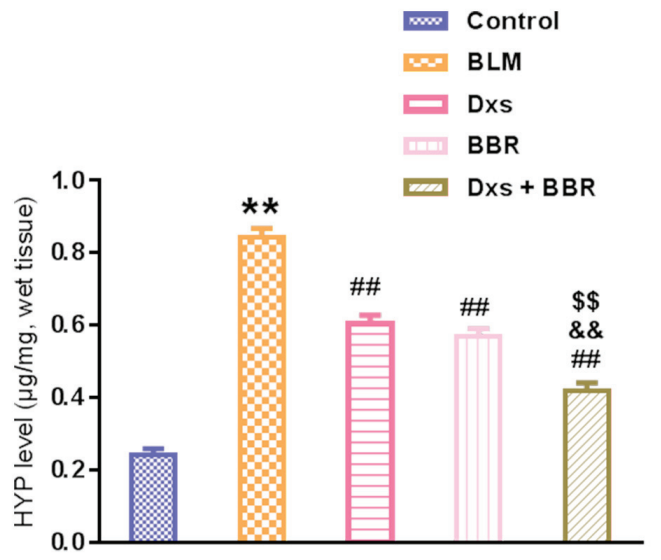

Figure 3. Quantitative analysis of Hyp levels in lung tissues from a rat mode with pulmonary fibrosis. Hyp levels in control animals, BLM-induced rats with PF and BLM-induced animals treated with Dxs, BBR or Dxs plus BBR were determined following 14 days of treatment. ${ }^{* *} \mathrm{P}<0.01$ vs. control ${ }^{\# \#} \mathrm{P}<0.01$ vs. $\mathrm{BLM}$; ${ }^{\$ \$} \mathrm{P}<0.01$ vs. Dxs; and ${ }^{\& \&} \mathrm{P}<0.01$ vs. BBR. BLM, bleomycin; Dxs, dexamethasone; BBR, berberine; Hyp, hydroxyproline.

BLM-induced PF at molecular levels and Smad2/3 signaling pathway activation served a role in this process.

Dxs plus BBR inhibits MMP2/9 expression. To study whether MMP2/9 are involved in the antifibrotic effect of Dxs plus BBR in BLM-treated rats, MMP2/9 expression was measured. ELISA assessment revealed that BLM significantly increased serum MMP2/9 levels in a time-dependent manner, with the highest increase observed on day 14 ( $\mathrm{P}<0.01$; Fig. 5A and $\mathrm{B})$. RT-qPCR and western blot analyses were conducted to assess MMP2/9 mRNA and protein expression in lung tissues on day 14 , respectively. It was observed that Dxs or BBR treatment significantly decreased MMP $2 / 9$ mRNA and protein levels compared with the BLM group $(\mathrm{P}<0.01)$. Dxs plus BBR treatment revealed to further significantly reduce MMP2/9 levels in serum or lung tissues compared with the Dxs and BBR single treatment groups (Fig. 5C-E).

Dxs plus BBR inhibits CXCL14 expression. To study whether CXCL14 was involved in the antifibrotic effect of Dxs plus BBR treatment, serum protein and lung tissue mRNA and protein levels of CXCL14 and CXCR4 were measured. As presented in Fig. 6A, a time-dependent increase in serum CXCL14 and CXCR4 levels was observed for the BLM group compared with the control $(\mathrm{P}<0.01)$, with the highest increase measured on day 14. BLM further significantly elevated CXCL14 and CXCR4 mRNA and protein levels in lung tissues on day 14 compared with the control group ( $\mathrm{P}<0.01$; Fig. 6B-D). Dxs or BBR treatment significantly decreased CXCL14 and CXCR4 levels in all samples compared with the BLM group $(\mathrm{P}<0.05)$. No marked differences were observed between these groups. In the Dxs plus BBR group, CXCL14 and CXCR4 levels in the serum and lung tissue were significantly lower compared with the Dxs and BBR single treatment groups $(\mathrm{P}<0.05)$.

Dxs plus BBR affects the Hh signaling pathway. To study whether the Hh signaling pathway was involved in the effect of Dxs plus BBR treatment, BLM-induced rats with PF were intraperitoneally injected with Dxs+BBR+purmorphamine and on day 14, CXCL14 mRNA and protein expression and Hyp content in lung tissues were assessed. Purmorphamine is used in Hh signaling pathway activation (14). It was observed that purmorphamine significantly enhanced CXCL14 mRNA, CXCL14 protein and Hyp levels in lung tissues compared with the Dxs plus BBR group $(\mathrm{P}<0.01$; Fig. $6 \mathrm{E}-\mathrm{G})$. The data suggested that inhibiting the Hh signaling pathway may be the primary mechanism by which Dxs plus BBR affect BLM-induced PF in rats.

\section{Discussion}

The BLM-induced PF model is widely used for diagnosing and treating PF and helping to explore underlying mechanisms (16). Treating rats with BLM via endotracheal injection caused a gradual increase of alveolar inflammation. On day 7 , inflammation started to increase, proliferation of fibroblasts was promoted and collagen production was increased. On day 14 , the alveolar structure disappeared and collagen was widely deposited, exhibiting first pathological indicators of PF. Hyp serves as one of the main components in collagen (17). Hyp is used to assess presence and extent of collagen deposition and is an accepted indicator for predicting drug efficacy in PF treatment (18). In the present study, a histological examination, Masson's trichrome staining and Hyp content in lung tissues were used to assess the rat PF model and its response to Dxs and BBR treatment on day 14. With Dxs or BBR intervention, lung damage and increases in Hyp content induced by BLM were markedly alleviated, confirming antifibrotic effects of BBR and Dxs at a histological level, which was in agreement with previous studies $(10,19)$. Combination of BBR and Dxs intervention exhibited stronger effects compared with the single intervention groups and lung damage and collagen deposition were reduced to a level similar to the control group. The results demonstrated that BBR plus Dxs treatment was an effective strategy to prevent PF.

The degree of PF was further assessed at molecular levels. Dxs or BBR were reported to prevent the Smad2/3 signaling pathway activation, which is a major determinant mechanism in promoting PF (11). Myofibroblast-derived collagen I/III and $\alpha$-SMA account for the provisional ECM accumulation (2). In the present study, it was assessed whether collagen I/III and $\alpha$-SMA were involved in the cooperative effect of BBR and Dxs treatment in regulating collagen deposition in the BLM-induced rat PF model and Smad2/3 signaling pathway activation was determined. A successful construction of a PF model was confirmed by severe collagen deposition and Smad2/3 signaling pathway activation in the BLM group, demonstrated by significant increases of collagen I/III, $\alpha$-SMA and $\mathrm{p}-\mathrm{Smad} 2 / 3$ levels. The present study further confirmed inhibitory effect of BBR or Dxs single treatment on the expression of these fibrotic makers. However, with BBR plus Dxs treatment, collagen I/III, $\alpha$-SMA and p-Smad $2 / 3$ levels significantly decreased further compared with the single treatments. The results demonstrated that the anti-fibrotic effect of BBR plus Dxs on BLM-induced PF were significantly enhanced when compared with BBR or Dxs alone with inhibition of Smad2/3 signaling pathway activation potentially the underlying mechanism. 


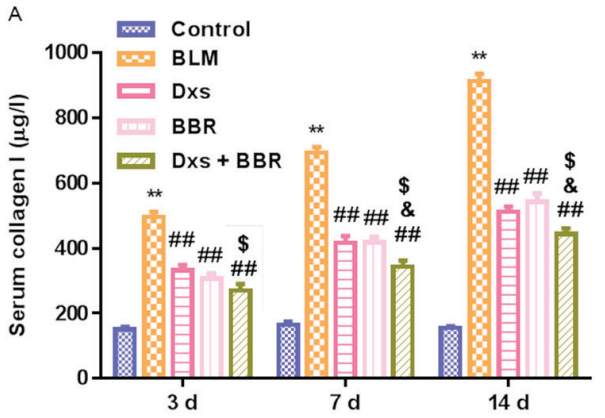

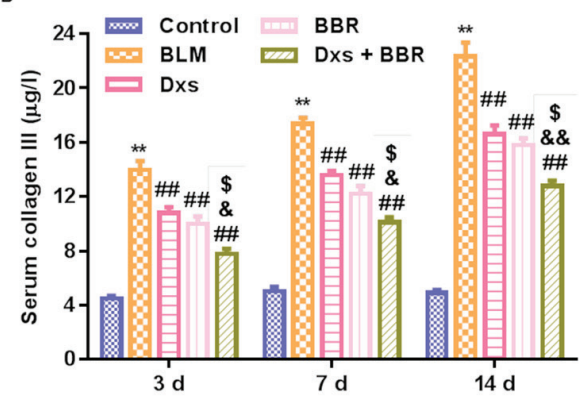

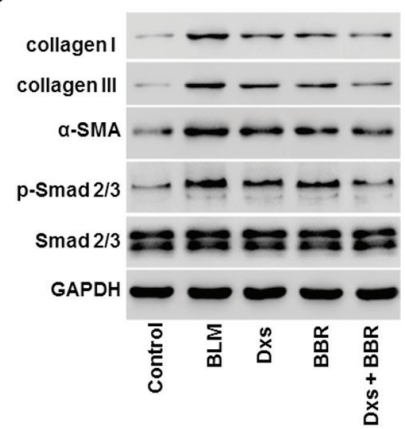

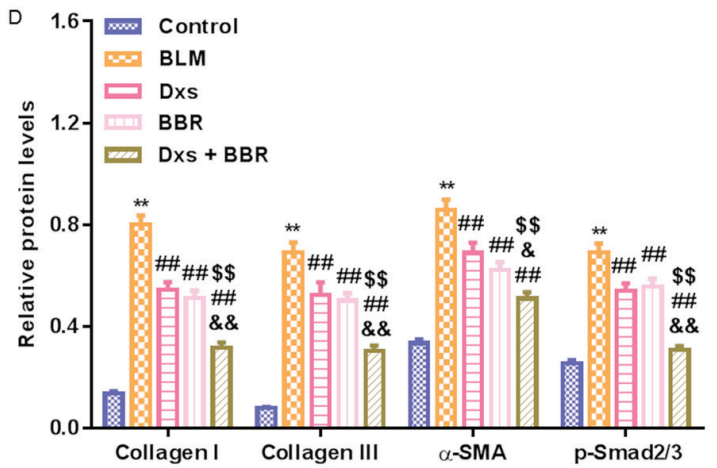

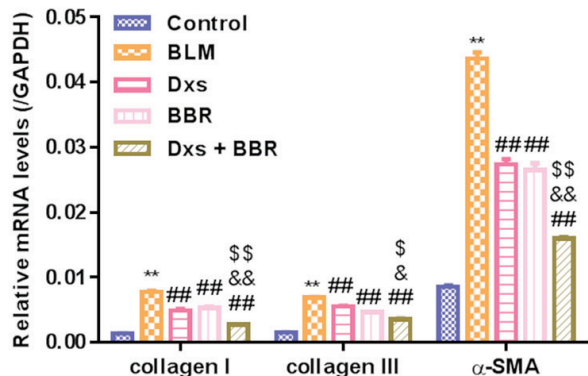

GAPDH was a loading control for collagen I/III, $\alpha$-SMA and Smad 2/3; Smad $2 / 3$ was used for $\mathrm{p}-\mathrm{Smad} 2 / 3$ normalization

Figure 4. Regulation of fibrogenesis-associated markers in a rat model with pulmonary fibrosis. A rat model was established, including control animals, BLM-induced PF rats and BLM-induced animals treated with Dxs, BBR or Dxs plus BBR. (A) Collagen I and (B) collagen III serum levels assessed by ELISA on days 3, 7 and 14 of treatment. Western blot (C) images and (D) quantitative analysis of lung tissues on day 14 evaluating collagen I/III, $\alpha-S M A, p-S m a d 2 / 3$, Smad2/3 and GAPDH. (E) Reverse transcription-qualitative polymerase chain reaction analysis of collagen I/III and $\alpha$-SMA in lung tissues on day 14 . GAPDH served as internal control for collagen I/III, $\alpha$-SMA and Smd2/3. Smd2/3 served as internal control for $\mathrm{p}-\mathrm{Smad} 2 / 3$. ${ }^{* *} \mathrm{P}<0.01$ vs. Control; ${ }^{\# \prime} \mathrm{P}<0.01$ vs. BLM; ${ }^{\$} \mathrm{P}<0.05$ and ${ }^{\$} \mathrm{P}<0.01$ vs. Dxs; and ${ }^{\&} \mathrm{P}<0.05$ and ${ }^{\&} \& \mathrm{P}<0.01$ vs. BBR. BLM, bleomycin; Dxs, dexamethasone; BBR, berberine; SMA, smooth muscle actin; p-, phosphorylated.

A

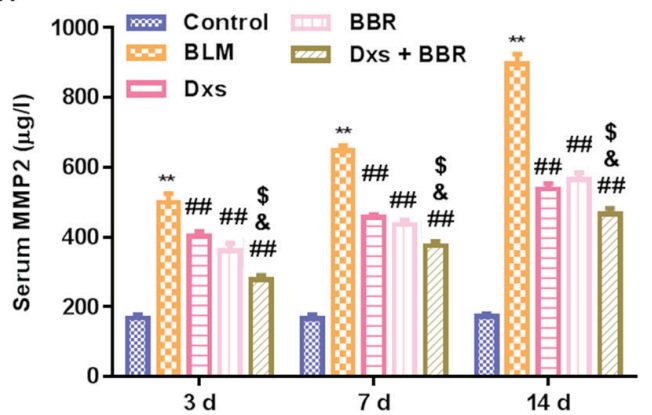

B

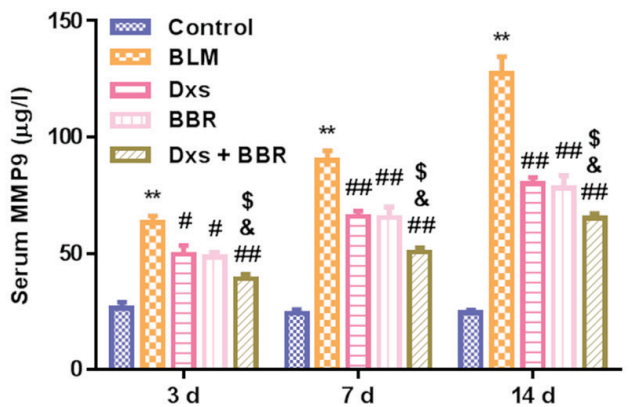

E
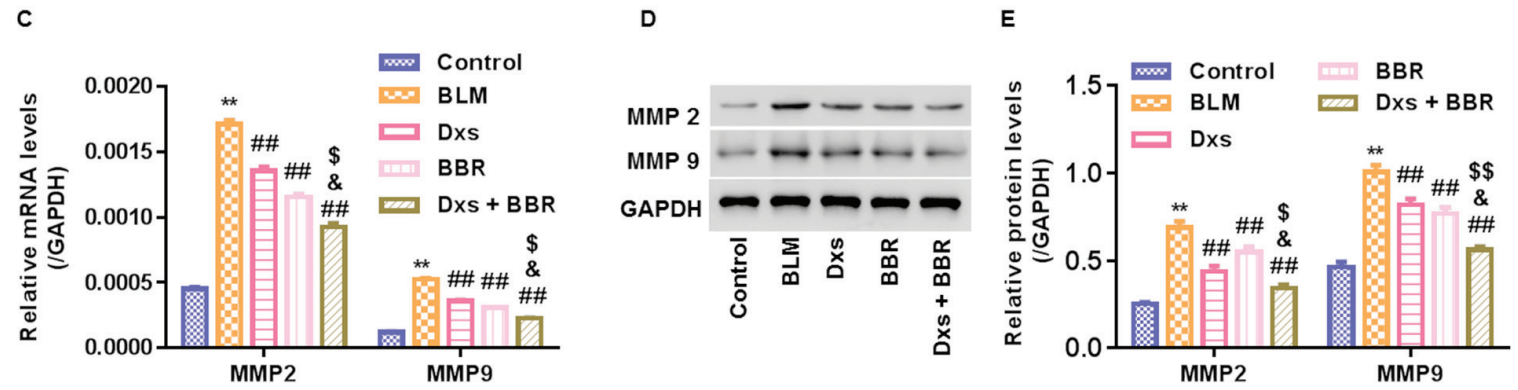

Figure 5. Regulation of MMP2 and MMP9 in a rat model with pulmonary fibrosis. A rat model was established, including control animals, BLM-induced PF rats and BLM-induced animals treated with Dxs, BBR or Dxs plus BBR. (A) MMP2 and (B) MMP9 serum levels assessed by ELISA on days 3, 7 and 14. (C) Reverse transcription-qualitative polymerase chain reaction analysis, (D) representative western blot images and (E) quantified western blot results of MMP2 and MMP9 in lung tissues on day. GAPDH served as internal control. ${ }^{* *} \mathrm{P}<0.01$ vs. Control; ${ }^{*} \mathrm{P}<0.05$ and ${ }^{\# \#} \mathrm{P}<0.01$ vs. BLM; ${ }^{\$} \mathrm{P}<0.05$ vs. Dxs; and ${ }^{\&} \mathrm{P}<0.05$ vs. BBR. BLM, bleomycin; Dxs, dexamethasone; BBR, berberine; MMP, matrix metalloproteinase. 
A

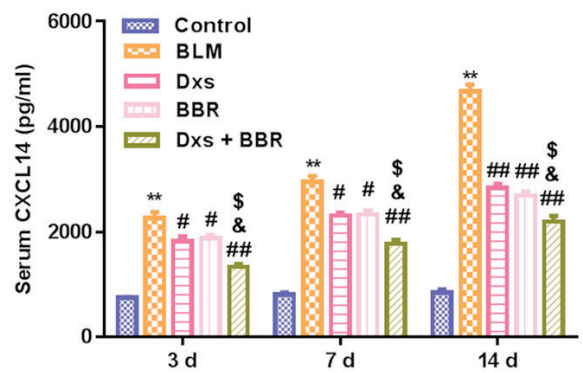

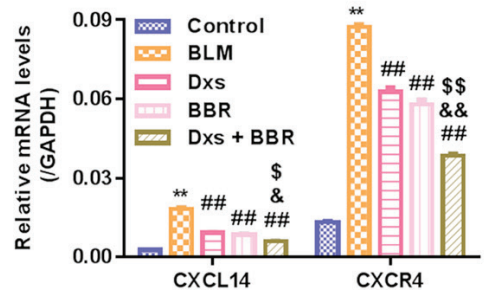

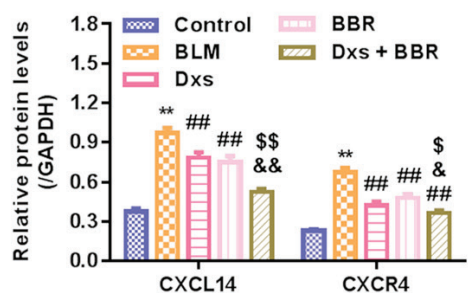

D

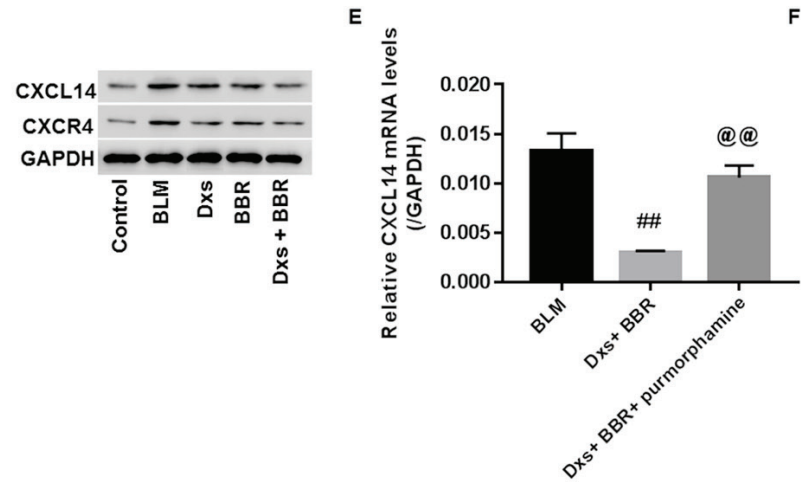

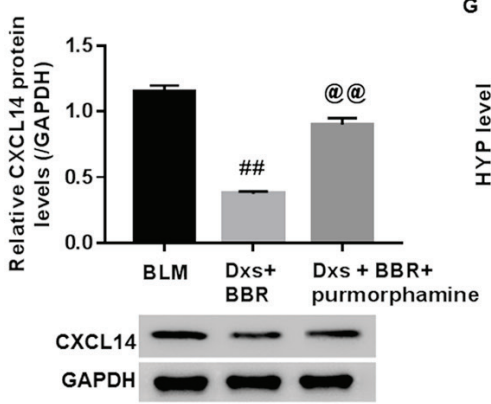

G

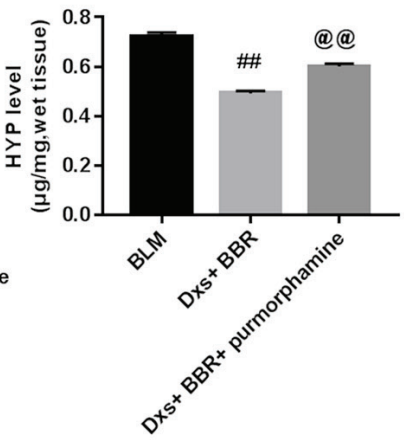

Figure 6. Regulation of CXCL14 in a rat model with pulmonary fibrosis. A rat model was established, including control animals, BLM-induced PF rats and BLM-induced animals treated with Dxs, BBR or Dxs plus BBR. (A) CXCL14 serum levels assessed by ELISA on days 3, 7 and 14. (B) Reverse transcription-qualitative polymerase chain reaction analysis, (C and D) western blot bands and quantification for CXCL14 and CXCR4 in lung tissues on day 14 . For the assessment of hedgehog signaling pathway activation, BLM-induced PF rats were treated with Dxs plus BBR or Dxs+BBR+purmorphamine. (E) Reverse transcription-qualitative polymerase chain reaction analysis, $(\mathrm{F})$ western blot images and quantification, and $(\mathrm{G}) \mathrm{Hyp}$ levels in lung tissues on day 14 . ${ }^{* *} \mathrm{P}<0.01$ vs. Control; ${ }^{\#} \mathrm{P}<0.05$ and ${ }^{\# \#} \mathrm{P}<0.01$ vs. BLM; ${ }^{\$} \mathrm{P}<0.05$ and ${ }^{\$ \$} \mathrm{P}<0.01$ vs. Dxs; ${ }^{\&} \mathrm{P}<0.05$ and ${ }^{\& \&} \mathrm{P}<0.01$ vs. BBR; ${ }^{\circledR} \mathrm{P}<0.01$ vs. Dxs+BBR. BLM, bleomycin; Dxs, dexamethasone; BBR, berberine; CXC, C-X-C motif; R, receptor; L, ligand; Hyp, hydroxyproline.

In a literature reported BLM-induced PF model, overall MMP2/9 expression in lung tissue determined by immunohistochemistry increases, with a peak on day 4 and decreases following day 7 , with distinctly higher levels observed until day 14 (20). In the present study, the levels of MMPs in serum and lung tissue was measured on day 3, 7 and 14 using ELISA and western bolt. By contrast, data demonstrated a time-dependent increase in MMPs in both serum and lung tissue of BLM-induced PF (Fig. 5). Dxs inhibits MMP2/9 expression in human lung cancer cells (21). Berberine (BBR) has further been reported to exhibit inhibitory effects on MMP2/9 in human airway smooth muscle HASMCs cells (22), breast cancer cells (23) and lung cancer A549 cells (23). However, data on the roles of BBR, Dxs or BBR plus Dxs in regulating MMP2/9 expression in BLM-treated rats are scarce. Findings of the current study suggested that with BBR or Dxs treatment, MMP2/9 levels were significantly reduced compared with the BLM group; however, the inhibitory effect was further significantly increased using combination treatment of BBR and Dxs. The results demonstrated an involvement of MMP2/9 in the prevention of PF. To further verify the activity of MMP2/9, a gelatin zymography assay may be performed in the future to measure levels of active and latent MMP2/9.

CXCL14 is upregulated during PF (24). Our previous study revealed that eliminating CXCL14 expression provides a therapeutic strategy for preventing mouse L929 fibroblasts from undergoing fibrogenesis (25). However, whether CXCL14 was involved in the anti-fibrotic effect of BBR or Dxs treatment, remains unknown. Data from the present study revealed that Dxs or BBR single treatment significantly reduced the levels of CXCL14 and its receptor CXCR4 compared with the BLM group. The combined used of BBR and Dxs induced significant decreases of CXCL14 and CXCR4 compared with the single treatment groups. This demonstrated an involvement of CXCL14 in the effectiveness of the combination treatment.

Activation of the $\mathrm{Hh}$ signaling pathway accelerates PF development and circulating CXCL14 has been used as a biomarker in assessing Hh signaling pathway activation (26). The current study further evaluated whether the Hh signaling pathway was involved in the antifibrotic function of Dxs plus BBR in the BLM-induced rat PF model. Data suggested that purmorphamine, an Hh signaling pathway activator, enhanced CXCL14 expression in the Dxs plus BBR group, indicating an activation of the Hh signaling pathway in the treatment process. In addition, it was observed that purmorphamine worsened rat $\mathrm{PF}$ in the BBR plus Dxs treatment group, as demonstrated by significantly increased Hyp content in lung tissues. The results suggested that Dxs plus BBR acted via the Hh signaling pathway to exert its antifibrotic effects in BLM-induced rat PF.

In conclusion, the results of the present study suggested that the combination of BBR and Dxs may have a cooperative effect on preventing BLM-induced rat PF. The antifibrotic mechanisms of BBR plus Dxs included downregulating MMP2/9 and CXCL14 levels and interrupting Smad2/3 and Hh signaling pathway activation. Dxs combined with BBR may represent an effective therapy to treat human PF. 


\section{Acknowledgements}

Not applicable.

\section{Funding}

The present study was supported by the Shanghai Municipal Health and Family Planning Commission 2016 (grant no. 20164Y0241).

\section{Availability of data and materials}

The datasets used and/or analyzed in the present study are available from the corresponding author on reasonable request.

\section{Authors' contributions}

HPL and YCQ designed the study. LL, QHL, FYL and YHS conducted experiments and performed data entry. LW, ZFW, WM, XLZ and SFZ were responsible for statistical analysis and data interpretation. LL, HPL, YCQ and XLZ prepared the manuscript. All authors read and approved the final manuscript.

\section{Ethical approval and consent to participate}

Experimental procedures and the Animal Use and Care protocols were approved by the Committee for Ethical Use of Animals of Baoshan District Hospital of Integrated Traditional Chinese and Western Medicine (Shanghai, China).

\section{Patient consent for publication}

Not applicable.

\section{Competing interests}

The authors declare that they have no competing interests.

\section{References}

1. Datta A,Scotton CJ and Chambers RC: Novel therapeutic approaches for pulmonary fibrosis. Br J Pharmacol 163: 141-172, 2011.

2. Wilson MS and Wynn TA: Pulmonary fibrosis: Pathogenesis, etiology and regulation. Mucosal Immunol 2: 103-121, 2009.

3. Wynn TA: Integrating mechanisms of pulmonary fibrosis. J Exp Med 208: 1339-1350, 2011.

4. Chakrabarti S and Patel KD: Matrix metalloproteinase-2 (MMP-2) and MMP-9 in pulmonary pathology. Exp Lung Res 31: 599-621, 2005.

5. Corbel M, Belleguic C, Boichot E and Lagente V: Involvement of gelatinases (MMP-2 and MMP-9) in the development of airway inflammation and pulmonary fibrosis. Cell Biol Toxicol 18: 51-61, 2002.

6. Liu YM, Nepali K and Liou JP: Idiopathic pulmonary fibrosis: Current status, recent progress, and emerging targets. J Med Chem 60: 527-553, 2017.

7. Keane MP, Strieter RM, Lynch JP III and Belperio JA: Inflammation and angiogenesis in fibrotic lung disease. Semin Respir Crit Care Med 27: 589-599, 2006.

8. Wuyts WA, Antoniou KM, Borensztajn K, Costabel U, Cottin V, Crestani B, Grutters JC, Maher TM, Poletti V, Richeldi L, et al: Combination therapy: The future of management for idiopathic pulmonary fibrosis? Lancet Respir Med 2: 933-942, 2014.
9. Li HP, Li X, He GJ, Yi XH and Kaplan AP: The influence of dexamethasone on the proliferation and apoptosis of pulmonary inflammatory cells in bleomycin-induced pulmonary fibrosis in rats. Respirology 9: 25-32, 2004

10. Chitra P, Saiprasad G, Manikandan R and Sudhandiran G: Berberine attenuates bleomycin induced pulmonary toxicity and fibrosis via suppressing NF- $\kappa \mathrm{B}$ dependant TGF- $\beta$ activation: A biphasic experimental study. Toxicol Lett 219: 178-193, 2013.

11. Chitra P, Saiprasad G, Manikandan R and Sudhandiran G: Berberine inhibits Smad and non-Smad signaling cascades and enhances autophagy against pulmonary fibrosis. J Mol Med (Berl) 93: 1015-1031, 2015.

12. Zhai L, Yan X, Wang H,Zhao N,Liang G and Tie XU: Experimental study on the therapeutic effect of penehyclidine hydrochloride plus dexamethasone on pulmonary fibrosis induced by paraquat in rats. Acta Academiae Med Xuzhou 30: 516-519, 2010.

13. Yang X, Wu L, Li G, Ran Q and Zhang L: Alphacalcidol combined with dexamethasone for reducing pulmonary fibrosis in mice and its mechanism. Xi Bao Yu Fen Zi Mian Yi Xue Za Zhi 33: 488-491, 2017 (In Chinese).

14. Chen MM, Bai HY, Zeng ZL and Neurology DO: The activation of the SHH pathway affects the cytoskeletal protein $\alpha$-tubulin and MAP-2 in stroke rat model. Zhong Feng Yu Shen Jing Ji Bing Za Zhi 32: 577-582, 2013 (In Chinese).

15. Livak KJ and Schmittgen TD: Analysis of relative gene expression data using real-time quantitative PCR and the 2(-Delta Delta C(T)) method. Methods 25: 402-408, 2001.

16. Tashiro J, Rubio GA, Limper AH, Williams K, Elliot SJ, Ninou I, Aidinis V, Tzouvelekis A and Glassberg MK: Exploring animal models that resemble idiopathic pulmonary fibrosis. Front Med (Lausanne) 4: 118, 2017.

17. Li $\mathrm{P}$ and $\mathrm{Wu} \mathrm{G}$ : Roles of dietary glycine, proline, and hydroxyproline in collagen synthesis and animal growth. Amino Acids 50: 29-38, 2018.

18. El-Kashef DH: Nicorandil ameliorates pulmonary inflammation and fibrosis in a rat model of silicosis. Int Immunopharmacol 64: 289-297, 2018

19. Chen XL, Xiao QM, Liu JJ, Xia-Hong HE and Ouyang B: PPAR $\gamma$ agonist and dexamethasone alleviate the pulmonary fibrosis induced by bleomycin in rats through upregulating glucocorticoid receptors. Xian Dai Sheng Wu Yi Xue Jin Zhan 23: 4609-4613, 2011 (In Chinese).

20. Kim JY, Choeng HC, Ahn C and Cho SH: Early and late changes of MMP-2 and MMP-9 in bleomycin-induced pulmonary fibrosis. Yonsei Med J 50: 68-77, 2009.

21. Roomi MW, Monterrey JC, Kalinovsky T, Niedzwiecki A and Rath M: Modulation of MMP-2 and MMP-9 by cytokines, mitogens and inhibitors in lung cancer and malignant mesothelioma cell lines. Oncol Rep 22: 1283-1291, 2009.

22. Liu SJ, Yin CX, Ding MC, Xia SY, Shen QM and Wu JD: Berberine suppresses in vitro migration of human aortic smooth muscle cells through the inhibitions of MMP-2/9, u-PA, AP-1, and NF-кB. BMB Rep 47: 388-392, 2014.

23. Kalaiarasi A, Anusha C, Sankar R, Rajasekaran S, John Marshal J, Muthusamy K and Ravikumar V: Plant Isoquinoline Alkaloid berberine exhibits chromatin remodeling by modulation of histone deacetylase to induce growth arrest and apoptosis in the A549 cell line. J Agric Food Chem 64: 9542-9550, 2016.

24. Ishii T, Nureki SI, Miyazaki E, Masuda D, Nishio S, Yamasue M, Fujisaki KH, Takenaka R, Takeo I, Masaru A and Kumamoto T: Elevated levels of BRAK/CXCL14 from patients with idiopathic pulmonary fibrosis. Am J Respiratory Crit Care Med 185: A5178, 2012.

25. Li L, Li Q, Wei L, Wang Z, Ma W, Liu F, Shen Y,Zhang S, Zhang X, $\mathrm{Li} \mathrm{H}$ and Qian Y: Chemokine (C-X-C motif) ligand 14 contributes to lipopolysaccharide-induced fibrogenesis in mouse L929 fibroblasts via modulating PPM1A. J Cell Biochem 120: 13372-13381, 2019.

26. Jia G, Chandriani S, Abbas AR, DePianto DJ, N'Diaye EN, Yaylaoglu MB, Moore HM, Peng I, DeVoss J, Collard HR, et al: CXCL14 is a candidate biomarker for Hedgehog signalling in idiopathic pulmonary fibrosis. Thorax 72: 780-787, 2017.

This work is licensed under a Creative Commons Attribution-NonCommercial-NoDerivatives 4.0 International (CC BY-NC-ND 4.0) License. 